\title{
PRISE EN CHARGE \\ DE L'HYPERPARATHYROIDIE PRIMAIRE : A PROPOS DE 25 CAS ET REVUE DE LA LITTÉRATURE
}

\author{
I. HARIGA, S. ZRIBI, K. KHAMASSI, O. BEN GAMRA, Y. SKOURI, M. BEN AMOR, \\ CH. MBAREK, A. EL KHEDIM \\ SERVICE D'ORL ET DE CHIRURGIE CERVICO-FACIALE, HÔPITAL HABIB THAMEUR \\ TUNIS, TUNISIE
}

\begin{abstract}
Objectif : Les auteurs rapportent leur expérience dans la prise en charge de l'hyperparathyroïdie primaire (HPTP) et comparent leurs résultats par rapport aux données de la littérature en discutant les avantages potentiels des différentes techniques chirurgicales.

Matériel et méthodes : Il s'agit d'une étude rétrospective à propos de 25 patients colligés sur 8 ans (1998-2005). Tous les patients ont bénéficié d'une échographie cervicale, d'un bilan rénal et d'un dosage de la calcémie et de la parathormonémie (PTH). Une TDM cervicale a été pratiquée dans 8 cas et une IRM dans 3 cas. Une scintigraphie à la tétrofosmine a été réalisée dans 10 cas, et à double isotope (thallium 201-technétium 99) dans 2 cas. Une cervicotomie avec exploration bilatérale systématique des 2 loges thyroïdiennes a été pratiquée dans tous les cas. En postopératoire, la surveillance a été clinique et biologique avec un recul moyen de 22 mois.

Résultats : La calcémie était élevée chez 23 patients et la PTH dans tous les cas. L'échographie a détecté une hypertrophie d'une glande parathyrö̈de chez 14 patients (56\%) et de 2 glandes chez 1 seul patient. La TDM cervicale a permis de localiser la lésion dans 5 cas. L'IRM était normale dans les 3 cas où elle a été pratiquée. La scintigraphie a montré une hyperfixation dans 8 cas. Une exérèse d'adénomes a été pratiquée dans $92 \%$ et une parathyroïdectomie subtotale dans $8 \%$ des cas. Le taux de guérison était de $88 \%$ après première intervention. Une reprise chirurgicale avec exérèse d'adénome a été pratiquée chez 2 patients (8\%). Par ailleurs aucune complication n'a été notée en postopératoire. Conclusion : La chirurgie parathyroïdienne classique donne de bons résultats avec des taux de guérison élevés et un taux de morbidité global faible. Ces résultats sont comparables avec ceux de la littérature. La chirurgie mini-invasive donne des résultats semblables mais impose des impératifs techniques, une imagerie performante et des patients rigoureusement sélectionnés.
\end{abstract}

Mots-clés : hypercalcémie, hyperparathyroïdie primaire, hyperparathormonémie, chirurgie parathyroïdienne

\footnotetext{
Objective : The authors report their experience in the management of primary hyperparathyroidism and compare their results to the literature with discussing the advantages of the different surgical techniques.

Materials and methods : We report a retrospective study about 25 patients treated between 1998 and 2005. All patients had cervical ultrasound, renal function, calcemia and parathyroid hormone (PTH) measurement. Cervical CT was performed in 8 cases and MRI in 3 cases. Tetrofosmin scintigraphy was performed in 10 cases and dual-isotope substraction scintigraphy (thallium 201-technetium 99) in 2 cases. Cervicotomy with systematic bilateral exploration was done in all cases. Postoperative follow-up was clinical and biological with a mean period of 22 months.

Results : Hypercalcemia was noted in 23 cases and elevated PTH level in all cases. Ultrasound showed hypertrophy of one parathyroid gland in 14 cases (56\%) and of 2 glands in one case. CT localized the lesion in 5 cases. MRI was normal in 3 cases. Scintigraphy detected hyperfixation in 8 cases. Exeresis of adenomas was performed in $92 \%$ and subtotal parathyroidectomy in $8 \%$ of cases. Recovery rate was $88 \%$ after first intervention. Two patients $(8 \%)$ were reoperated with exeresis of adenomas. On the other hand, no complications were noted postoperatively.

Conclusion : Classical parathyroid surgery achieves good results with high recovery rate and low morbidity global rate. These results are comparable with those of the literature. Minimally invasive surgery provides similar results but imposes technical requirements, high performance imaging and rigorously selected cases.
}

Keywords : hypercalcemia, primary hyperparathyroidism, hyperparathormonemia, parathyroid surgery 


\section{INTRODUCTION}

L'hyperparathyroïdie primaire (HPTP) est une maladie caractérisée par une sécrétion inappropriée de la parathormone (PTH) pouvant être responsable d'une hypercalcémie. Actuellement la chirurgie représente son seul traitement curatif. La localisation chirurgicale de toutes les glandes parathyroïdes permet en fait d'obtenir un taux de guérison supérieur à $95 \%$ avec un taux de complication inférieur à $2 \%(1,2)$. Cette approche nécessite cependant une incision large et une dissection bilatérale afin d'identifier des glandes qui se révèlent le plus souvent normales. En effet, les HPTP sont dues à la présence d'un adénome unique dans 85 à $90 \%$ des cas (3) Ceci explique l'intérêt croissant pour les approches mini invasives, devenues actuellement possibles grâce aux progrès d'imagerie et aux dosages peropératoires de la PTH. Dans ce travail, nous rapportons notre expérience ainsi qu'une revue de la littérature concernant la prise en charge de l'HPTP.

\section{MATÉRIEL ET MÉTHODES}

II s'agit d'une étude rétrospective à propos de 25 patients traités et suivis sur 8 ans (1998-2005) et ayant une HPTP. Tous les patients ont bénéficié d'un examen ORL complet, d'une échographie cervicale, d'un bilan rénal et d'un dosage de la calcémie et de la parathormonémie. Une TDM cervicale a été réalisée dans 8 cas et une IRM dans 3 cas. Une scintigraphie à la tétrofosmine a été pratiquée dans 10 cas, et à double isotope (thallium 201-technétium 99) dans 2 cas. Le traitement chirurgical a consisté en une approche classique avec exploration bilatérale systématique des 2 loges thyroïdiennes après repérage et dissection des 2 nerfs récurrents. Un dosage de la calcémie et de la parathormonémie a été pratiqué en postopératoire dans tous les cas. Le recul moyen était de 22 mois.

\section{RESULTATS}

L'âge moyen était de 56,5 ans (26-74 ans) et le sex-ratio 0,13 . Le délai moyen de consultation était de 16 mois.

Les signes d'appel étaient par ordre décroissant :

- uronéphrologiques (44\%): dominés par les coliques néphrétiques en rapport avec des lithiases.

- ostéoarticulaires (40\%): il s'agissait essentiellement de fracture pathologique (Fig. 1) ou de tumeur brune (Fig. 2). - digestifs (36\%): en rapport avec un ulcère gastro-duodénal.

- cardiovasculaires et généraux (28\%).

L'HPTP était asymptomatique et de découverte fortuite au cours d'un bilan systématique chez 1 seul patient. L'examen ORL trouvait une tuméfaction basi-cervicale chez 8 patients (double dans 2 cas) dont 3 seulement correspondaient à des adénomes parathyroïdiens en peropératoire, les autres nodules étaient thyroïdiens.
La calcémie était élevée chez 23 patients avec une moyenne de 2,87 mmol/l (2,63-3,16 mmol/l). La calciurie réalisée chez 22 patients était élevée dans 16 cas. La parathormonémie était élevée dans tous les cas avec une moyenne de $405 \mathrm{pg} / \mathrm{ml}$ (92-1057 pg/ml). La fonction rénale était normale chez tous les patients.

L'échographie a détecté une hypertrophie d'une glande parathyroïde chez 14 patients (56\%) et de 2 glandes chez 1 seul patient. La TDM cervicale, pratiquée chez 8 patients a permis de localiser la lésion dans 5 cas. L'IRM n'a pas révélé de lésions dans les 3 cas où elle a été pratiquée. La scintigraphie à la tétrofosmine a montré une hyperfixation parathyroïdienne unique dans 7 cas (Fig. 3). Celle à double isotope a été contributive chez 1 patient en montrant l'atteinte d'une parathyroïde.

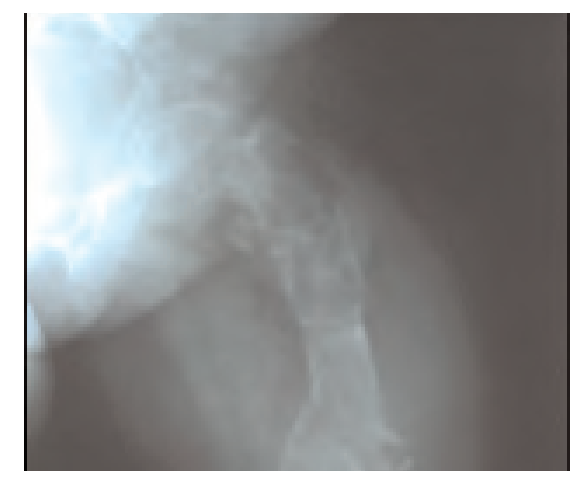

Fig. 1 : Fracture pathologique du fémur

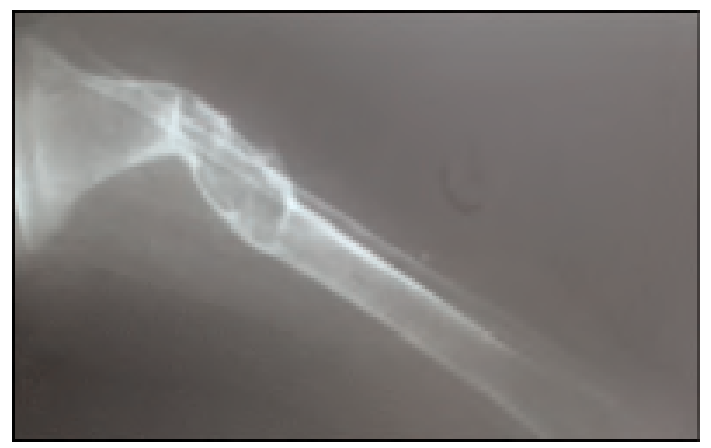

Fig. 2 : Tumeur brune du tibia

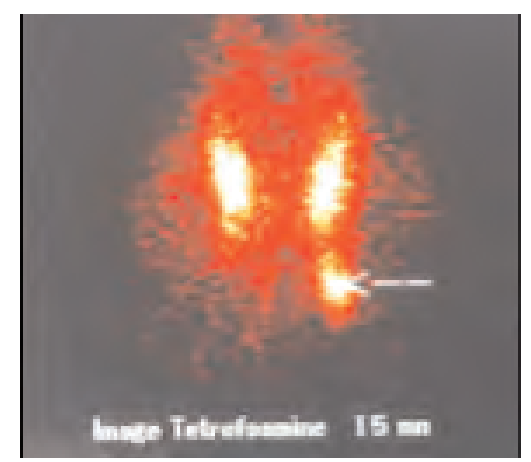

Fig. 3: Scintigraphie à la tétrofosmine: Adénome parathyroïdien inférieur gauche 
La cervicotomie exploratrice a révélé une hypertrophie parathyroïdienne unique chez 22 patients (88\%) (Fig. 4)

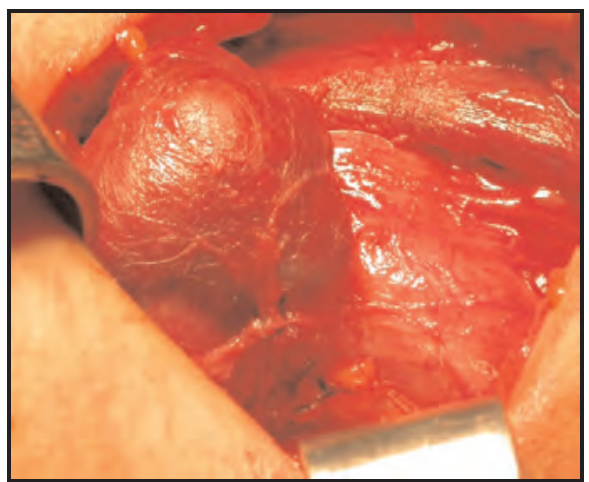

Fig. 4 : Adénome parathyroïdien (Vue peropératoire)

dont l'examen extemporané aprés exérèse, a confirmé la nature adénomateuse dans 20 cas et hyperplasique dans 1 cas. Une atteinte double a été notée chez un patient. Celui-ci a bénéficié de l'exérèse des 2 adénomes. Par ailleurs, une hyperplasie des 4 parathyroïdes a été notée chez 2 patients, imposant une parathyroïdectomie subtotale. Une lobo-isthmectomie a été associée dans 14 cas devant la présence de nodules thyroïdiens. La cervicotomie a été blanche chez 2 patients malgré une exploration minutieuse de la loge thyroïdienne, de la région prétrachéale et du médiastin antéro-supérieur. Chez ces 2 patients, un complément d'exploration par une scintigraphie au MIBI et une IRM cervico-thoracique a permis de déceler l'adénome au niveau du médiastin antéro-supérieur chez un patient (en latéro-trachéal droit) et au niveau du médiastin postéro-supérieur chez l'autre (à la hauteur de D1). Ils ont été réopérés et l'adénome a été localisé et enlevé dans les deux cas. (Tableau I)

\begin{tabular}{|c|c|c|}
\hline Constatations peropératoires & Geste chirurgical & Anatomopathologie \\
\hline \multirow[t]{2}{*}{ Hypertrophie d'une glande $(\mathrm{N}=22)$} & Exérèse + Lobo-isthm ectomie : 14 & \\
\hline & Exérèse : $\quad 8$ & Adénome \\
\hline Hypertrophie de 2 glandes & Exérèse & 2 Adénomes \\
\hline Hyperplasie & Parathyroïdectomie subtotale & Hyperplasie \\
\hline
\end{tabular}

Tableau I : Geste chirurgical et anatomopathologie

Au total, 24 adénomes chez 23 patients ont été notés après examen anatomopathologique, et dont le siège inférieur droit était le plus fréquent (Tableau II).

\begin{tabular}{|c|c|}
\hline Siège & Nombre \\
\hline P III droite & 12 \\
P III gauche & 5 \\
P IV droite & 2 \\
P IV gauche & 2 \\
Sous-isthmique & 1 \\
Médiastin antéro-sup & 1 \\
Médiastin postéro-sup & 1 \\
\hline Total & $\mathbf{2 4}$ \\
\hline
\end{tabular}

P III : Glande Parathyroïde inférieure

P IV : Glande Parathyroïde supérieure

Tableau II : Sièges des adénomes
En postopératoire, une PTH et une calcémie élevées ont persisté chez un patient ayant subi une parathyroïdectomie subtotale. L'imagerie et la scintigraphie à la tétrofosmine étaient négatives.

Par ailleurs, une récidive de l'hyperparathyroïdie a été notée chez une patiente, 3 ans après l'exérèse d'un adénome parathyroïdien inférieur droit. La scintigraphie au $\mathrm{MIBI}$ a montré une hyperfixation polaire supérieure droite. La patiente a été reprise avec succès et bonne évolution en postopératoire.

Par ailleurs, aucune hypocalcémie persistante ni paralysie récurrentielle n'ont été notées chez tous les patients. Sur le plan général, on a noté une régression des signes cliniques et une régénération osseuses chez les patients ayant initialement une tumeur brune (Fig. 5).

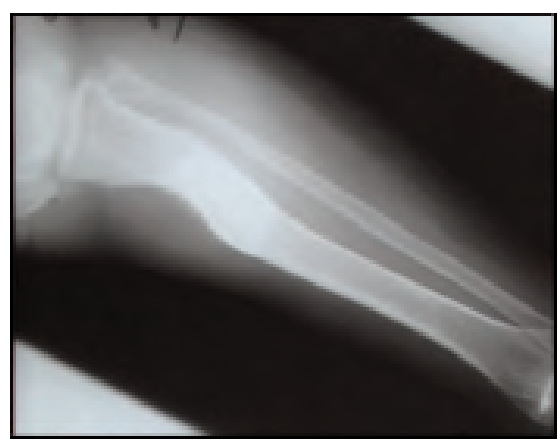

Fig. 5 : Ossification de la tumeur brune après traitement chirurgical de I'HPTP

\section{DISCUSSION}

Le traitement actuel de l'HPTP repose sur la chirurgie. L'indication opératoire est formelle dans les formes symptomatiques ou avec présence de troubles biologiques ou de signes radiologiques en rapport avec l'affection (96\% de nos patients). En cas d'HPTP asymptomatique, l'hypercalcémie, l'atteinte rénale et la diminution de la densité osseuse représentent les principales indications de la chirurgie. Le choix de la voie d'abord varie selon les auteurs.

Les explorations bilatérales pour l'HPTP obtiennent un des taux de guérisons les plus élevées et s'accompagnent d'un taux de morbidité et de mortalité très faible. Cependant, la dissection étendue afin d'identifier des glandes qui s'avèrent le plus souvent normales a sussité le recours à une approche unilatérale moins invasive et plus rapide. Ce type de chirurgie a été décrit en 1982 par Tibblin (4) mais n'a connu de réel intérêt que grâce aux progrès de l'imagerie médicale. En effet, la scintigraphie utilisant le methoxyisobutyl isonitrile (MIBI) marqué au technétium-99 et l'échographie à haute résolution ont permis la localisation préopératoire des adénomes parathyroïdiens avec une sensibilité supérieure à $85 \%(3,5)$. L'IRM, indiquée surtout en deuxième intention en cas de scintigraphie non concluante, a une sensibilité de $73 \%$ et qui augmente jusqu'à $92 \%$ si elle est associée à une scintigraphie au MIBI (6). Par 
ailleurs, le dosage peropératoire par méthodes radio-immunologiques du taux de parathormone intacte (PTHi) a permis de confirmer la résection de toutes les glandes hypersécrétantes et de limiter l'exploration peropératoire.

Ainsi de nouvelles techniques de parathyroïdectomie se sont ainsi développées: abord unilatéral (7), chirurgie radioguidée $(8,9,10)$, chirurgie mini-invasive ouverte $(11,12)$, chirurgie mini-invasive vidéo-assistée ou endoscopique $(13,14,15)$. Toutes ces techniques sont pratiquées moyennant des incisions limitées et sont ciblées sur une seule glande parathyroïde. Par ailleurs, la chirurgie vidéo-assistée permet l'exploration complète de tous les éléments anatomiques de la région rétrothyroïdienne, depuis le pédicule thyroïdien supérieur jusqu'au médiastin postéro-supérieur. Cependant, les avantages potentiels de ces approches restent toujours discutés.

\section{Abord classique ou chirurgie mini-invasive?}

Une approche classique bilatérale, quand elle est réalisée par un chirurgien expérimenté, permet d'obtenir un taux de guérison élevé pouvant atteindre 98,2\% (16) voire 100\% (17). Ce taux était de $88 \%$ dans notre série après première intervention. Les rares échecs de cette chirurgie sont observés chez des patients présentant des lésions multiglandulaires, des glandes surnuméraires ou ectopiques, ou en cas d'exceptionnels cancers parathyroïdiens.

Des séries de résection parathyroïdienne utilisant une approche unilatérale ont obtenu des résultats similaires avec des taux de guérison variant entre 95 et $100 \%$ $(18,19,20)$. Ces séries sont cependant de tailles moins importantes et portent le plus souvent sur des patients soigneusement sélectionnés. En effet, des antécédents de chirurgie thyroïdienne, l'existence d'un goitre, la suspicion de lésions multiglandulaires ou de cancer parathyroïdien, et l'absence de localisation de l'adénome parathyroïdien en préopératoire constituent très souvent des contre-indications à cette approche et imposent d'emblée un abord classique.
Par ailleurs, un des avantages potentiels d'une approche unilatérale est la réduction du taux de morbidité grâce à un abord direct de la lésion entraînant un risque moindre de lésion parathyroïdienne et d'hypocalcémie persistante. En cas d'approche bilatérale, le taux d'hypocalcémies postopératoires définitives est de $1 \%$, celui de lésions récurrentielles est de $0,2 \%$ (16). Ces taux sont nuls dans notre série et sont comparables à ceux des approches unilatérales $(15,18)$. Cependant, l'absence de dissection systématique du nerf récurrent en cas d'abord direct exposerait à un risque accru de lésion nerveuse justifiant pour certains auteurs un abord classique systématique.

Sur le plan anesthésique, la plupart des explorations unilatérales sont réalisables sous anesthésie locale pure ou bloc cervical, contrairement aux approches bilatérales. Ceci permettra de réduire les risques d'anesthésie chez les patients âgés ou tarés et de diminuer la durée de l'intervention, de l'hospitalisation et de convalescence. Néanmoins, ceci nécessite une collaboration totale de la part du patient, condition qui n'est pas toujours assurée dans la majorité des cas.

Enfin, sur le plan esthétique, les résultats sont habituellement meilleurs après approche unilatérale. Bien que cet aspect n'ait pas fait l'objet d'étude spécifique, l'incision de taille réduite et la dissection moindre permet d'obtenir un meilleur résultat esthétique et offre de meilleurs conditions en cas de réintervention (18).

\section{CONCLUSION}

La prise en charge de l'hyperparathyroïdie primaire repose sur la chirurgie qui constitue le seul traitement curatif de cette maladie, et qui donne de bons résultats avec des taux de guérison élevés et un taux de morbidité global faible indépendamment de la voie d'abord et de la technique utilisée. La chirurgie mini-invasive impose des impératifs techniques, une imagerie performante et des patients rigoureusement sélectionnés.

\section{REFERENCES}

(1) Chen H, Zeiger MA, Gordon TA, Udelsman R. Parathyroidectomy in Maryland: effects of an endocrine center. Surgery 1996;120:948-953.

(2) Van Heerden JA, Grant CS. Surgical treatment of primary hyperparathyroidism: an institutional perspective. World J Surg 1991;15:688-692.

(3) Denham DW, Norman J. Cost-effectiveness of preoperative sestamibi scan for primary hyperparathyroidism is dependent solely upon the surgeon's choice of operative procedure. J Am Coll Surg 1998:186:293-305.

(4) Tibblin S, Bondeson AG, Ljungberg O. Unilateral parathyroidectomy in hyperparathyroidism duet o a single adenoma. Ann Surg 1982;195:245-252. (5) Chapuis YD, Fulla Y, Bonnichon PH, Tarla E, Abboud B, Pitre J et al. Values of ultrasonography, sestamibi scintigraphy and intraoperative measurement of 1 84 PTH for unilateral neck exploration of primary hyperparathyroidism. World $J$ Surg 1996;20:835-840

(6 Saeed S, Yao M, Philip B, Blend M. Localizing hyperfunctioning parathyroid tissue: MRI or nuclear study or both? Clinical Imaging 2006;30:257-265.

(7) Chapuis YD, Richard B, Fulla Y, Bonnichon PH, Tarla E, Icard P. Chirurgie de l'hyperparathyroïdie primaire par abord unilateral sous anesthésie locale et dosage peropératoire de la PTH 1-84. Chirurgie 1993-1994;119:121-124. (8) Norman J, Chheda H. Minimally invasive parathyroidectomy facilitated by intra-operative nuclear mapping. Surgery 1997;122:998-1004.

(9) Burkey SH, Van Heerden JA, Farley DR, Thompson GB, Grant CS, Curlee KJ. Will directed parathyroidectomy utilizing the gamma pribe or intra-operative parathyroid hormone assay replace bilateral cervical exploration as the preferred operation for primary hyperparathyroidism? World J Surg 2002;26:914-920. (10) Lokey J, Mondragon-Sanchez A, Salazar Navarro F, Pattou F, Carnaille B,
Huglo D, Proye C. Chirurgie radioguidée de l'hyperparathyroïdie primaire. Expérience de 75 observations. Ann Chir 2001:126:535-540.

(11) Udelsman R, Donovan PI, Sokoll LJ. One hundred consecutive minimally invasive parathyroid explorations. Ann Surg 2000;232:331-339.

(12) Ikeda Y, Takami H, Tajima G, Sasaki Y, Takayama J, Kurihara H, et al. Direct mini-incision parathyroidectomy. Biomed Pharmacother 2002;56:14s-17s.

(13) Gagner M. Endoscopic parathyroidectomy. Br J Surg 1996:83:875

(14) Suzuki S, Fukushima T, Ami H, Asahi S, Takenoshita S. Video-assisted parathyroidectomy. Biomed Pharmacother 2002;56:18s-21s.

(15) Henry JF, Sebag F, Maweja S, Hubbard J, Misso C, Da Costa V, Tadivet L. Place actuelle de la chirurgie vidéo-assistée dans le traitement de l'hyperparathyroïdie primaire. Ann Chir 2003:128:379-384.

(16) Low R, Katz A. Parathyroidectomy via bilateral exploration: a retrospective review of 866 patients. Head Neck 1998;20:583-587.

(17) Mbarek M, Cherif L, Annabi H, Mbarek Ch, Cherif MR, Zribi S, et al. Fractures pathologiques multiples révélatrices d'hyperparathyroïdie. A propos de 3 cas. J Tun ORL 2004:12:59-62.

(18) Inabnet WB, Biertho L. Chirurgie parathyroïdienne dirigée: une série de 100 patients consécutifs. Ann Chir 2002;127:751-756.

(19) Inabnet WB, Fulla Y, Richard B, Bonnichon P, Icard P, Chapuis Y. Unilateral neck exploration under local anesthesia : the approach of choice for asymptomatic primary hyperparathyroidism. Surgery 1999;126:1004-1009.

(20) Flynn MB, Bumpous JM, Schill K, McMasters KM. Minimally invasive radioguided parathyroidectomy. J Am Coll Surg 2000;191:24-31. 